\title{
Whole Wiskott-Aldrich syndrome protein gene deletion identified by high throughput sequencing
}

\author{
XIANGLING HE, RUNYING ZOU, BING ZHANG, YALAN YOU, YANG YANG and XIN TIAN \\ Department of Hematology and Oncology of Children's Medical Center, Hunan Provincial People's Hospital/The \\ First Affiliated Hospital of Hunan Normal University, Changsha, Hunan 410005, P.R. China
}

Received July 21, 2016; Accepted May 3, 2017

DOI: $10.3892 / \mathrm{mmr} .2017 .7416$

\begin{abstract}
Wiskott-Aldrich syndrome (WAS) is a rare $\mathrm{X}$-linked recessive immunodeficiency disorder, characterized by thrombocytopenia, small platelets, eczema and recurrent infections associated with increased risk of autoimmunity and malignancy disorders. Mutations in the WAS protein (WASP) gene are responsible for WAS. To date, WASP mutations, including missense/nonsense, splicing, small deletions, small insertions, gross deletions, and gross insertions have been identified in patients with WAS. In addition, WASP-interacting proteins are suspected in patients with clinical features of WAS, in whom the WASP gene sequence and mRNA levels are normal. The present study aimed to investigate the application of next generation sequencing in definitive diagnosis and clinical therapy for WAS. A 5 month-old child with WAS who displayed symptoms of thrombocytopenia was examined. Whole exome sequence analysis of genomic DNA showed that the coverage and depth of WASP were extremely low. Quantitative polymerase chain reaction indicated total WASP gene deletion in the proband. In conclusion, high throughput sequencing is useful for the verification of WAS on the genetic profile, and has implications for family planning guidance and establishment of clinical programs.
\end{abstract}

\section{Introduction}

Wiskott-Aldrich syndrome (WAS) is a rare X-linked recessive primary immunodeficiency disease recognized by symptoms including eczema, thrombocytopenia, immune deficiency and bloody diarrhea (1). It is also known as eczema-thrombocytopenia-immunodeficiency syndrome, in line with Aldrich's

Correspondence to: Dr Runying Zou, Department of Hematology and Oncology of Children's Medical Center, Hunan Provincial People's Hospital/The First Affiliated Hospital of Hunan Normal University, 61 Jiefang West Road, Changsha, Hunan 410005, P.R. China

E-mail: hexiangl@163.com

Key words: Wiskott-Aldrich syndrome, X-linked recessive inheritance, primary immunodeficiency disease, next generation sequencing, polymerase chain reaction original description in 1954 (2). The molecular defects of WAS were discovered in 1994 by Derry et al (3), who isolated the pathogenic gene by positional cloning. The gene that encodes the WAS protein (WASP) is located in the short arm of X chromosome (XP11.22-11.23) and is $\sim 9 \mathrm{~kb}$, containing 12 exons and encoding 502 amino acids.

Epidemiological studies have demonstrated that the incidence of neonatal WAS in the developed countries is $1 / 1,000,000-10 / 1,000,000$ (4), and WAS is caused by a mutation in the WASP gene. WASP is a hematopoietic system-specific intracellular signal transduction molecule, which is proline rich, expressed only in hematopoietic cell lines. It is well documented that WASP gene mutations affect the expression of WASP, thereby resulting in disorders in the response of non-red blood cells to external stimuli, which in turn leads to issues in signal transduction and cytoskeleton dysfunction. This further influences the number, size and aggregation of platelets, hence leading to defects in lymphocytes migration, signal transduction and immunological synapse formation (5).

WASP has been revealed to be critical for cell-signaling (6), actin polymerization (7), synapse formation (8), cell/cell interaction (9), chemotaxis (10), and Treg function (11). As a result, clinical manifestations of patients with alterations of the WASP gene show a great heterogeneity. Categorized by the mutations in the WASP gene, WAS can be divided into typical classic WAS, X-linked thrombocytopenia (XLT) and X-linked neutropenia (XLN). Classic WAS is characterized by triad of thrombocytopenia/micro-platelets, recurrent infections, and eczema. The milder XLT variant behaves predominantly as thrombocytopenia, sometimes occurring intermittently. Patients with congenital neutropenia but without the clinical findings characteristic of WAS or XLT are classified as XLN (12).

Different mutations of WASP could lead to the heterogenicity of WAS in patients. In addition to WASP, WASP interacting proteins have been demonstrated to be involved in the onset of WAS (13-15). Disruption of the binding of WASP to its interacting proteins could also lead to patients with clinical features of WAS, in whom the WASP gene sequence and mRNA levels are normal (16-18). The binding of WASP with its interacting proteins have been confirmed by crystallography (19-21).

It is not difficult to diagnose WAS according to the clinical features; however, as the clinical manifestations of patients demonstrate a great heterogeneity, definite diagnosis 
is required in many cases. Previously, Sanger sequencing of the WASP gene has been applied for the confirmation of WAS diagnosis $(22,23)$, as WASP gene mutations account for the majority of WAS cases. However, as WAS may be caused by mutations of other WASP interacting proteins, other genes may be involved; therefore, performing only WASP gene detection may lead to misdiagnosis. Therefore, it is necessary to identify multiple genes simultaneously. In the present study, next generation sequencing was performed on a WAS patient so as to give the definite conclusion and provide evidence for therapy based on the genetic profile.

\section{Patients and methods}

Patient data. A 5-month old male pediatric patient with thrombocytopenia for 3 months and rash for 2 months was admitted to Hunan Provincial People's Hospital (Changsha, China). His mother was a 25 -year old gravida 1 para 1 healthy woman without familial diseases. This child was identified to have thrombocytopenia in a local hospital 35 days after birth, when he was hospitalized due to a lung infection. Gamma globulin protein shock treatment was ineffective and his platelet levels maintained at (30-36) $\times 10^{9} / 1$. When he was 2 months old, repeated eczematous rashes appeared. His parents confirmed the family history. The present study was approved by the ethics committee of Hunan Provincial People's Hospital, and informed consents were obtained from the parents of the participant and healthy controls.

Investigation of WASP-interacting proteins. To investigate potential proteins that may interact with WASP, the online server STRING (www.string-db.org/) was applied. This server provides a database of known and predicted protein-protein interactions (24). The network analysis was performed strictly according to the user documentation. The search was initiated using the Single Protein by Name/Identifier selection of the website.

Next-generation sequencing (NGS). For specimen preparation, genomic DNA from $2 \mathrm{ml}$ peripheral blood was extracted from the trio of the proband following the instruction of BloodGen Midi kit (CWBIO, Beijing, China) and the DNA was sheared by sonication. The sheared genomic DNA was then hybridized with NimbleGen 2.0 probe sequence capture array (Roche Diagnostics, Basel, Switzerland). The captured DNA was firstly applied for exonic DNA enrichment (Roche Diagnostics) and the libraries were then tested for enrichment by quantitative polymerase chain reaction (qPCR) using the Agilent Bioanalyzer 2100 (Agilent Technologies, Inc., Santa Clara, USA). The samples were thereby sequenced on an Illumina Hiseq2500 system (Illumina, Inc., San Diego, CA, USA).

Raw image files were processed by the BclToFastq (Illumina, Inc.) for base calling and raw data generating. The low-quality variations were filtered out using quality score $\geq 20$ (Q20). The sequencing reads were aligned to the NCBI human reference genome (hg19) using the Burrows-Wheeler Alignment tool (25). The Genome Analysis Toolkit (26) was used to analyzed single nucleotide polymorphism and insertion-deletion of the sequences. The genes that encoded WASP interacting proteins predicted by STRING were analyzed in detail.
PCR amplification. PCR amplification was performed using whole genome DNA of the proband and a 25-year-old female healthy control. Whole genome DNA was extracted from peripheral blood using DNAzol ${ }^{\mathrm{TM}}$ BD Reagent (Thermo Fisher Scientific, Inc., Waltham, MA, USA). Specific primers covering most exons of the WASP gene were designed using Primer premier 5.0 software (Premier Biosoft International, Palo Alto, CA, USA). DNA polymerase was purchased from Takara Bio, Inc. (Otsu, Japan). The primer sequences and relative product lengths are presented in Table I. The PCR conditions were as follows: Initial denaturation at $94^{\circ} \mathrm{C}$ for $5 \mathrm{~min}$, followed by 40 cycles of denaturation at $94^{\circ} \mathrm{C}$ for $30 \mathrm{sec}$, annealing at $60^{\circ} \mathrm{C}$ for $30 \mathrm{sec}$, elongation at $72^{\circ} \mathrm{C}$ for $30 \mathrm{sec}$, and extension at $72^{\circ} \mathrm{C}$ for $5 \mathrm{~min}$. PCR products were applied to $1 \%$ agarose gel electrophoresis and analyzed by image acquisition and analysis system (Tanon Science and Technology Co., Ltd., Shanghai, China).

qPCR amplification. qPCR amplification was performed using whole genome DNA extracted from peripheral blood of the mother and a 25-year-old female healthy control using DNAzol $^{\mathrm{TM}}$ BD Reagent (Thermo Fisher Scientific, Inc.). Specific fluorescence quantitative primers were designed for the $\mathrm{N}$ terminal, middle and $\mathrm{C}$ terminal of the WASP gene with Primer premier 5.0 software. The primer sequences and relative product lengths are presented in Table II. The primers were synthetized by Sangon Biotech Co., Ltd. (Shanghai, China). The PCR conditions were as follows: Initial denaturation at $95^{\circ} \mathrm{C}$ for $1 \mathrm{~min}$, followed by 40 cycles of denaturation at $95^{\circ} \mathrm{C}$ for $15 \mathrm{sec}$, annealing at $60^{\circ} \mathrm{C}$ for $40 \mathrm{sec}$ and elongation at $68^{\circ} \mathrm{C}$ for $30 \mathrm{sec}$. For each sample, two parallel reactions were performed and tyrosine-protein kinase ABL1 (ABL1) served as a reference gene. Homogenization of the PCR products were firstly performed using the output of ABL. Amplification efficiency was calculated according to the comparative $\mathrm{Cq}$ method (27) by drawing the standard curve of each pair of primers. The copy number ratio of each truncated gene was calculated by comparing with that of the control.

Statistical analysis. Statistical analysis was performed using SPSS software version 17.0 (SPSS, Inc., Chicago, IL, USA). The rations of the truncated WASP genes of the mother were calculated to that of the control and then expressed as mean \pm standard deviation. The value was then compared with the estimated value of 0.5 using single-sample Student's t-test. $\mathrm{P}<0.05$ was considered to indicate a statistically significant difference.

\section{Results}

Clinical features of the patient. Following hospitalization, routine examinations of the blood, urine and stools were performed. Physical examination revealed scattered needlepoint to small grain-sized papules mixed with a few pinpoint-sized bleeding points across the whole body. Parts of the eruptions were accompanied by a little secretion, with periauricular as the most serious. Purulent secretion was identified in the left ear. The liver was enlarged $6 \mathrm{~cm}$ below the right costal margin and the spleen could be palpated $2 \mathrm{~cm}$ under the left costal margin. Blood routine examination revealed that the 
Table I. Primers used for specific truncated WASP gene detection for the proband and a healthy control by polymerase chain reaction amplification.

\begin{tabular}{llc}
\hline Primer & \multicolumn{1}{c}{ Sequence (5'-3') } & Product length (bp) \\
\hline WAS-1F & TCTAAGCAGTCAAGTGGAGGAG & 930 \\
WAS-1R & ATCTGGATGAGTCTTTGGTTCTG \\
WAS-2F & GAGCCTCAACTTCCTAAGACTAGA \\
WAS-2R & TCAGCCATCTACCGCCAATC \\
WAS-3F & TACCTCCATGACCATCCAACA \\
WAS-3R & CCATCCTTCCATTCACTCAGC \\
WAS-4F & TTCCATAACTCCTGCCTATACTCA \\
WAS-4R & CACTGACCAACTCCTGACTGA \\
WAS-5AF & TCACTCAGTCCTTATGGGAGCACCT \\
WAS-5AR & TCAAACAGATGGGGCTGATGTCACT \\
WAS-6F & TTAACCAGACAGGAAGCAAT \\
WAS-6R & CTTGAGTGAAGAGAACTGAGA & 380 \\
\end{tabular}

F, forward; R, reverse; WASP, Wiskott-Aldrich syndrome protein.

Table II. Primers used for specific truncated WASP gene detection for the mother and a healthy control by quantitative polymerase chain reaction amplification.

\begin{tabular}{llc}
\hline Primer & \multicolumn{1}{c}{ Sequence (5'-3') } & Product length (bp) \\
\hline WAS-1QF & AAGACCTTGTGGCTACCCCT & 144 \\
WAS-1QR & AGCACACAGCCCCACAATGCTC & 151 \\
WAS-3QF & GTCAATGAGCCAACCACCCTA & 136 \\
WAS-3QR & TTCTTATCAGCTGGGCTAGGTC \\
WAS-5QF & CTAAGCCCTCTGTGCTGATCCC & 136 \\
WAS-5QR & GGCTCTGCTTCTCTTCTGCATCAC & \\
\hline
\end{tabular}

F, forward; R, reverse; WASP, Wiskott-Aldrich syndrome protein.

white blood cell count was $14.28 \times 10^{9} / 1$, the neutrophil count was $7.42 \times 10^{9} / 1$, hemoglobin was $116 \mathrm{~g} / 1$, the platelet count was $26 \times 10^{9} / 1$, the thrombocytocrit was $0.01 \%$, and the platelet mean volume was $5.8 \mathrm{fl}$. Stool and urine routine tests indicated that liver function, renal function, myocardial enzyme, blood glucose, electrolytes, coagulation and C-reactive protein all were roughly normal. The erythrocyte sedimentation rate was $37 \mathrm{~mm} / \mathrm{h}$. The fecal occult blood test was negative. Examination of immunoglobulin revealed that immunoglobulin (Ig)A was $0.76 \mathrm{~g} / 1$, IgG was $9.73 \mathrm{~g} / 1$ [after intravenous gammaglobulin (IVIG)] and $\operatorname{IgM}$ was $5.88 \mathrm{~g} / \mathrm{l}$; all were higher than healthy limits. In addition, total $\mathrm{IgE}$ was within the normal range (11.36 IU/ml). Lymphocyte subsets detection demonstrated that cluster of differentiation (CD) $3^{+} \mathrm{T}$ cells accounted for $38.4 \%$ of lymph, $\mathrm{CD}^{+} \mathrm{CD} 4$ helper/inducer T cells were $32.9 \%$ of lymph, and the percentage of $\mathrm{CD}^{+} \mathrm{CD}^{+}$suppressor/cytotoxic $\mathrm{T}$ cells was $1.3 \%$, which were a little lower than that of normal values. The percentage of CD3 ${ }^{-} \mathrm{CD}^{2} 6^{+} \mathrm{CD}^{-} 6^{+}$natural killer cells and $\mathrm{CD}^{-}{ }^{-} \mathrm{CD} 19^{+} \mathrm{B}$ cells were 16.2 and $35.9 \%$ respectively, which were a little higher compared with normal limits.

Respiratory virus antigen examination (influenza A, influenza B, respiratory tract virus, adenovirus, parainfluenza virus 1 ,
2 and 3) and Torch analysis were negative. cytomegalovirus-DNA was $<1.00 \mathrm{E}+03$ copies and blood culture was negative. A chest $\mathrm{X}$-ray revealed scattered patchy shadow in both lungs with a fuzzy edge, whereas no obvious abnormalities in cardiophrenic angle were identified. A cranial plain X-ray was normal and an abdominal ultrasound revealed hepatosplenomegaly. A bone marrow examination revealed that proliferation of bone marrow was active. A large number of megakaryocytes were observed, and there was megakaryocyte mature hindrance in cell classification.

Acute feverish and shortness of breath occurred 3 days after the child was admitted to hospital. He was transferred to the intensive care unit for treatment as he was diagnosed severe pneumonia, respiratory failure and sepsis. In line with the above symptoms, the child was clinically diagnosed as typical WAS. As his condition aggregated, his parents abandoned treatment and the child was followed up.

WASP-interacting proteins identification results. In order to gain insight into the proteins that interact with WASP, the online server STRING was used. The predictive result is represented in Fig. 1. Together, there were 10 proteins that 


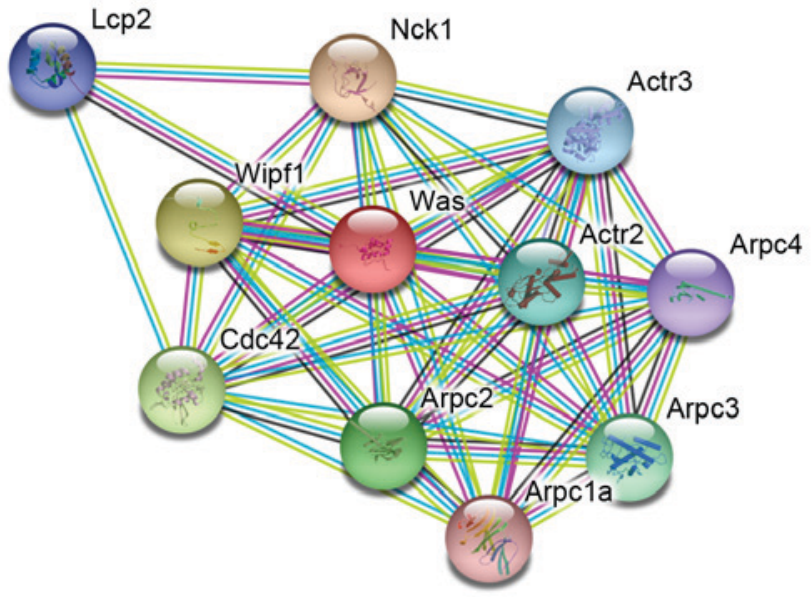

Figure 1. Analytic result of WASP-interacting proteins obtained by using the online database STRING. WAS, Wiskott-Aldrich syndrome; WASP, Wiskott-Aldrich syndrome protein; Arpc, actin-related protein 2/3 complex subunit; Wipf1, WAS/WASL-interacting protein family member 1; Nck1, cytoplasmic protein Nck1; Lcp2, lymphocyte cytosolic protein 2; Cdc42, cell division control protein 42 homolog; Actr2, actin-related protein 2.

were considered to be associated with WASP directly or indirectly. The results indicated that $>$ one proteins are enrolled in the interaction of WASP; NGS may reveal novel insights into the genetic profile of WAS.

$N G S$ results. To confirm the diagnosis of WAS and unravel the underlying genetic mutations in WASP and other genes, genomic DNA from the proband and his parents underwent NGS. A total of $8.7 \mathrm{G}$ clean data was obtained from the three samples. The average of GC content was $42.92 \%$ and the Q20 was $>95 \%$. Quality control files demonstrated that the data was reliable and adequate for further analysis. Coverage and mean depth for the child, his mother and his father were $0.24 / 0.6$, $1 / 32.9$ and $1 / 48.4$, respectively. No mutation that could support the determination of other disease besides WAS was identified. The results indicated that the WASP gene was deviant or even totally lost in the proband, and confirmed the diagnosis of WAS.

PCR results indicate the whole WASP gene is lost in the proband. To assess the deletion information in the WASP gene in the proband, PCR was performed. As presented in Fig. 2, no bands were observed in all the lanes $(1,3,5,7,9$ and 11) of PCR products from the patient, whereas relative bands were observed in all lanes $(2,4,6,8,10$ and 12) from that of the healthy control. The results indicated that the WASP gene in the proband was totally lost.

$q P C R$ results of the mother of the proband. As the father was healthy, the lost mutation could only be inherited from his mother or caused de novo. To reveal the etiology so as to guide reproduction to this family, the whole genomic DNA of the mother underwent qPCR analysis. As presented in Fig. 3, the ratios of the target truncated WASP genes to that of the control all were $\sim 0.5$, with a mean of $0.58 \pm 0.10$. There were significant differences in the mean ratio value for the mother $(\mathrm{t}=1.453$, $\mathrm{P}=0.284$ ). The results demonstrated that the WASP gene of the mother was heterozygous, and the mutation of the proband was inherited from his mother.

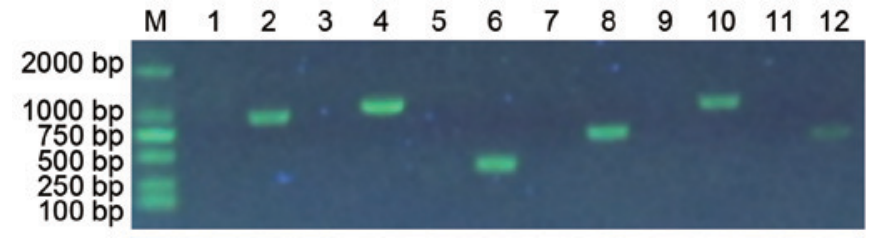

Figure 2. PCR result indicate the whole WASP gene is lost in the proband Lanes $1,3,5,7,9$, and 11 are PCR products from the proband, while lanes $2,4,6,8,10$, and 12 are PCR products with the corresponding primers from a healthy control. The lanes $2,4,6,8,10$, and 12 are products of WAS-1F and WAS-1R, WAS-2F and WAS-2R, WAS-3F and WAS-3R, WAS-4F and WAS-4R, WAS-5AF and WAS-5AR, WAS-6F and WAS-6R primers, respectively. PCR, polymerase chain reaction; WAS, Wiskott-Aldrich syndrome; F, forward; R, reverse.
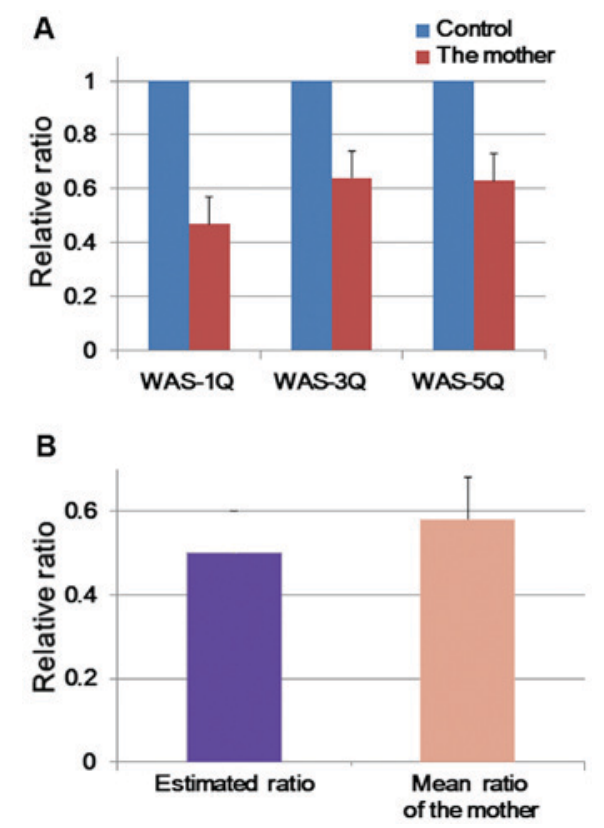

Figure 3. qPCR results indicate that the mother of the proband was heterozygous. (A) The relative ratio of the qPCR WAS products of specific sequences from the mother were compared with a healthy control.(B) Data are expressed as the mean \pm standard deviation. No significant differences were detected. qPCR, quantitative polymerase chain reaction; WAS, Wiskott-Aldrich syndrome.

\section{Discussion}

At present, $>300$ kinds of WAPS gene mutations have been reported, which are distributed in the whole WAS gene, and are more concentrated in exon 1-4s, 7 and 10 (28). Six sites are regarded as mutational hotspots, accounting for $\sim 25 \%$ of all mutational sites, including three splice site mutations and three point mutations in the coding region (29). The mutation type of the WAS gene determines the expression of WASP, which is closely associated with the clinical phenotype of WAS. A missense mutation in exons 1-3 usually leads to the XLT phenotype, loss of or truncated WASP expression of the 10th exon could induce typical WAS, and missense mutations in GTPase binding domain (L270P, S272P and 1294T) could lead to XLN (30).

Peng et al (31) observed that from January 1991 to October 2013, there were 12 articles focusing on gene diagnosis of WAS including 54 case studies. Mutations consisting of 
missense, nonsense, splicing site and insertion/deletion mutations were identified; however, no whole exon deletion was observed. In a retrospective study containing 50 case studies from 40 pedigrees by Catucci et al (32), missense and fusion mutations manifested as WASP positive expression, whereas nonsense mutations, deletion mutations and insertional mutagenesis led to WASP-negative expression. Among the 10 cases of deletion mutations, 3 cases were large fragment deletions, and 1 case was whole exon deletion. In the present study, the patient had whole exon 1-12 deletion, which is rare. To the best of our knowledge, this is the first case of whole exon deletion reported in China, and the second case in the world.

WAS is a combined immunodeficiency disease, with different degrees of humoral and cellular immune deficiency. As the abnormal immune function of WAS is gradual, the immune deficiency symptoms increase with age. Therefore, immunological examination results vary markedly. In most cases, it is reported that serum IgM decreases, IgG only slightly decreases or remains normal, $\operatorname{IgA}$ and $\operatorname{IgE}$ elevate, and $\mathrm{T}$ and $\mathrm{B}$ cell proliferation is reduced in $50 \%$ patients in vitro (4). In the present study, T cell counts reduced whereas B lymphocyte numbers increased, which is consistent with other studies $(4,33)$. However, there was difference in the humoral immunity result. Serum IgE was normal and $\operatorname{IgA}$ and IgG levels were elevated, which may be associated with intravenous Ig therapy. IgM levels increased significantly, which may be associated with repeated intrauterine and postnatal infection by combing with liver and spleen enlargement. This indicates that the immune level of the patient may be associated with age, disease course, infection and other factors that affect disease progression. Therefore, WAS diagnosis should not only be based on the outcome of immunodetection.

Autoimmune diseases often occur in children with WAS, and in the United States and the European population the incidence is as high as 40 72\% (32). Autoimmune hemolytic anemia, vasculitis, arthritis and kidney disease are the most common symptoms. In a retrospective study, Imai et al (33) demonstrated that there is no statistically significant difference between WASP negative and positive groups in autoimmune disease incidence (22 vs. $26 \%, \mathrm{P}>0.05$ ) (33). Five cases were with $\operatorname{Ig}$ A nephropathy and the age of onset was rather late, from 10 to 20 years old. In addition, 5 out of the 50 reviewed patients were complicated by tumors, and they all were WASP negative. In the present study, neither autoimmune or tumor diseases were identified; this may be associated with the young age of the patient and the short duration of the disease. Zhao et al (34) suggested that the clinical phenotype of WAS evaluation should follow the dynamic principle. In line with their opinion, whether this patient's disease may be associated autoimmune diseases and tumors requires further follow-up observation.

Therapeutic methods of WAS should be planned according to clinical severity, duration, mutations of the WASP gene and WASP expression. Therefore, it is necessary to identify the etiology on genetic profile. Routinely, Sanger sequencing is performed to detect mutational situation in the WASP gene. However, as WAS may be caused by WASP-interacting proteins, it is essential to identify the mutations in these proteins simultaneously so as give systemic identification. In view of this, the present study performed whole exome sequencing for this patient.
Before sequencing, the STRING online database was used to identify WASP-interacting proteins, and the result demonstrated that 10 proteins are included. Among the 10 proteins, actin-related protein 2/3 complex subunit (Arpc)1a, Arpc2, Arpc3, Arpc4, Actr 2 and Actr3 are associated with the Arp2/3 protein complex, which has been implicated in the control of actin polymerization in cells and are conserved through evolution (35-38). In addition, they have been demonstrated to interact with Cortactin (35). The protein WAS/WASL-interacting protein family member 1 (Wipf1) is reported to interact with WASP, Cortactin, and cytoplasmic protein NCK1 $(13,14)$. NCK1 is linked to glucose tolerance and insulin signaling within certain tissues, and serves important roles in insulin signaling and the c-Jun N-terminal kinase signaling pathway (39). Cdc42 is involved in diverse cellular functions, including cell morphology, migration, endocytosis and cell cycle progression (40). The functions of these proteins are associated with that of WASP $(6,9,10)$, indicating that it is necessary for detecting the status of these genes. The sequencing results indicated that there were no abnormal WASP-interacting protein coding genes, but only whole loss of the WASP gene. No novel mutations in WASP-interacting protein coding genes were identified, indicating that NGS was indispensable for WAS diagnosis.

Supportive treatment and antibiotic prophylaxis are required for typical WAS; however, it is necessary to perform IVIG and platelet transfusion and splenectomy. As for typical WAS, hematopoietic stem cell transplantation is the most effective method for early stage of onset. Without hematopoietic stem cell transplantation, WAS patients will eventually die because of infections, bleeding, malignant tumors and other complications (41). As the patient in the present study has severe WAS, hematopoietic stem cell transplantation is the first choice for the treatment; however, his parents terminated treatment because of economic reasons. To date, this patient is followed up. As the genetic profile had been perfectly revealed, the result serve a significant role for family planning guidance for the parents.

In conclusion, this proband has a typical clinical phenotype of WAS; however, at Sanger sequencing was insufficient to determine the pathogenesis, therefore NGS was performed. NGS indicated whole exon deletion of the WASP gene, and the result was further confirmed by Sanger sequencing. Although once reported, whole exon deletion of this gene is very rare. The one reported case was from Japan and this case is in China, which may indicate that whole exon deletion is prone to occur in Asians. WAS is a rare immunodeficiency disease; due to the diversity of WASP gene mutations, the clinical manifestations are very different, and therapeutic methods of WAS should be planned according to clinical severity. The traditional diagnostic approach for WAS is Sanger sequencing, which is flawed as WASP mutation types are varied and other genes in addition to WASP could also induce WAS. Therefore, it is necessary to perform NGS for pathogenic identification and subsequent family planning guidance. The present study aimed to identify mutations in other genes beside the WASP gene to demonstrate the powerful functionality of NGS both for genotyping and for treatment scheme planning; however, this study identified only whole exon deletion. However, in line with the gene deletion type, it is presumed that the prognosis will be very poor, thereby hematopoietic stem cells transplantation is the first 
choice for treatment, which highlights the importance of NGS as a diagnostic tool.

\section{Acknowledgements}

The present study was supported by the Planned Projects of Hunan Provincial Science and Technology Department (grant no. 2013FJ6028) and the Scientific Fund of Healthy and Family Planning Commission of Hunan Province (grant no. C2013-023).

\section{References}

1. Lemahieu V, Gastier JM and Francke U: Novel mutations in the Wiskott-Aldrich syndrome protein gene and their effects on transcriptional, translational and clinical phenotypes. Hum Mutat 14 54-66, 1999.

2. Aldrich RA, Steinberg AG and Campbell DC: Pedigree demonstrating a sex-linked recessive condition characterized by draining ears, eczematoid dermatitis and bloody diarrhea. Pediatrics 13: 133-139, 1954.

3. Derry JM, Ochs HD and Francke U: Isolation of a novel gene mutated in Wiskott-Aldrich syndrome. Cell 78: 635-644, 1994.

4. Ochs HD and Thrasher AJ: The Wiskott-Aldrich syndrome. J Allergy Clin Immunol 117: 725-739, 2006.

5. Blundell MP, Worth A, Bouma G and Thrasher AJ: The Wiskott-Aldrich syndrome: The actin cytoskeleton and immune cell function. Dis Markers 29: 157-175, 2010.

6. Westerberg LS, Dahlberg C, Baptista M, Moran CJ, Detre C, Keszei M, Eston MA, Alt FW, Terhorst C, Notarangelo LD and Snapper SB: Wiskott-Aldrich syndrome protein (WASP) and N-WASP are critical for peripheral B-cell development and function. Blood 119: 3966-3974, 2012.

7. Adriani M, Aoki J, Horai R, Thornton AM, Konno A, Kirby M, Anderson SM, Siegel RM, Candotti F and Schwartzberg PL: Impaired in vitro regulatory $\mathrm{T}$ cell function associated with Wiskott-Aldrich syndrome. Clin Immunol 124: 41-48, 2007.

8. Wada T, Schurman SH, Garabedian EK, Yachie A and Candotti F: Analysis of T-cell repertoire diversity in Wiskott-Aldrich syndrome. Blood 106: 3895-3897, 2005.

9. Maillard MH, Cotta-de-Almeida V, Takeshima F, Nguyen DD, Michetti P, Nagler C, Bhan AK and Snapper SB: The Wiskott-Aldrich syndrome protein is required for the function of CD4(+) CD25(+) Foxp3(+) regulatory T cells. J Exp Med 204: 381-391, 2007.

10. Yarar D, To W, Abo A and Welch MD: The Wiskott-Aldrich syndrome protein directs actin-based motility by stimulating actin nucleation with the Arp2/3 complex. Curr Biol 9: 555-558, 1999.

11. Davis BR and Candotti F: Revertant somatic mosaicism in the Wiskott-Aldrich syndrome. Immunol Res 44: 127-131, 2009.

12. Ochs HD: Mutations of the Wiskott-Aldrich syndrome protein affect protein expression and dictate the clinical phenotypes. Immunol Res 44: 84-88, 2009.

13. Luthi JN, Gandhi MJ and Drachman JG: X-linked thrombocytopenia caused by a mutation in the Wiskott-Aldrich syndrome (WAS) gene that disrupts interaction with the WAS protein (WASP)-interacting protein (WIP). Exp Hematol 31: 150-158, 2003.

14. Bender M, Stritt S, Nurden P, van Eeuwijk JM, Zieger B, Kentouche K, Schulze H, Morbach H, Stegner D, Heinze KG, et al: Megakaryocyte-specific Profilin1-deficiency alters microtubule stability and causes a Wiskott-Aldrich syndrome-like platelet defect. Nat Commun 5: 4746, 2014.

15. Sasahara Y: WASP-WIP complex in the molecular pathogenesis of Wiskott-Aldrich syndrome. Pediatr Int 58: 4-7, 2016.

16. Lanzi G, Moratto D, Vairo D, Masneri S, Delmonte O, Paganini T, Parolini S, Tabellini G, Mazza C, Savoldi G, et al: A novel primary human immunodeficiency due to deficiency in the WASP-interacting protein WIP. J Exp Med 209: 29-34, 2012.

17. Fried S, Matalon O, Noy E and Barda-Saad M: WIP: More than a WASp-interacting protein. J Leukoc Biol 96: 713-727, 2014.

18. Ramesh N, Massaad MJ, Kumar L, Koduru S, Sasahara Y, Anton I, Bhasin M, Libermann T and Geha R: Binding of the WASP/ N-WASP-interacting protein WIP to actin regulates focal adhesion assembly and adhesion. Mol Cell Biol 34: 2600-2610, 2014.

19. St-Jean M, Izard T and Sygusch J: A hydrophobic pocket in the active site of glycolytic aldolase mediates interactions with Wiskott-Aldrich syndrome protein. J Biol Chem 282: 14309-14315, 2007.
20. Ti SC, Jurgenson CT, Nolen BJ and Pollard TD: Structural and biochemical characterization of two binding sites for nucleation-promoting factor WASp-VCA on Arp2/3 complex. Proc Natl Acad Sci USA 108: E463-E471, 2011.

21. Jurgenson CT and Pollard TD: Crystals of the Arp2/3 complex in two new space groups with structural information about actin-related protein 2 and potential WASP binding sites. Acta Crystallogr F Struct Biol Commun 71: 1161-1168, 2015.

22. Wada T, Itoh M, Maeba H, Toma T, Niida Y, Saikawa Y and Yachie A: Intermittent X-linked thrombocytopenia with a novel WAS gene mutation. Pediatr Blood Cancer 61: 746-748, 2014.

23. Takimoto $\mathrm{T}$, Takada $\mathrm{H}$, Ishimura $\mathrm{M}$, Kirino $\mathrm{M}$, Hata $\mathrm{K}$, Ohara O, Morio T and Hara T: Wiskott-Aldrich syndrome in a girl caused by heterozygous WASP mutation and extremely skewed $\mathrm{X}$-chromosome inactivation: A novel association with maternal uniparental isodisomy 6. Neonatology 107: 185-190, 2015.

24. Szklarczyk D, Franceschini A, Wyder S, Forslund K, Heller D, Huerta-Cepas J, Simonovic M, Roth A, Santos A, Tsafou KP, et al: STRING v10: Protein-protein interaction networks, integrated over the tree of life. Nucleic Acids Res 43 (Database Issue): D447-D452, 2015.

25. Li H and Durbin R: Fast and accurate short read alignment with Burrows-Wheeler transform. Bioinformatics 25: 1754-1760, 2009.

26. Wassner AJ, Cohen LE, Hechter E and Dauber A: Isolated central hypothyroidism in young siblings as a manifestation of PROP1 deficiency: Clinical impact of whole exome sequencing. Horm Res Paediatr 79: 379-386, 2013.

27. Livak KJ and Schmittgen TD: Analysis of relative gene expression data using real-time quantitative PCR and the 2(-Delta Delta C(T)) method. Methods 25: 402-408, 2001.

28. Thrasher AJ: New insights into the biology of Wiskott-Aldrich syndrome (WAS). Hematology Am Soc Hematol Educ Program: 132-138, 2009.

29. Jin Y, Mazza C, Christie JR, Giliani S, Fiorini M, Mella P, Gandellini F, Stewart DM, Zhu Q, Nelson DL, et al: Mutations of the Wiskott-Aldrich syndrome protein (WASP): Hotspots, effect on transcription and translation, and phenotype/genotype correlation. Blood 104: 4010-4019, 2004.

30. Ancliff PJ, Blundell MP, Cory GO, Calle Y, Worth A, Kempski H, Burns S, Jones GE, Sinclair J, Kinnon C, et al: Two novel activating mutations in the Wiskott-Aldrich syndrome protein result in congenital neutropenia. Blood 108: 2182-2189, 2006.

31. Peng F, Nong G, Jiang M, Liu X, Liu H and Li Y: Clinical features, genotype and phenotype analysis and literature review of Wiskott-Aldrich syndrome. J Applied Clin Pediatr 29: 675-679, 2014.

32. Catucci M, Castiello MC, Pala F, Bosticardo M and Villa A: Autoimmunity in wiskott-Aldrich syndrome: An unsolved enigma. Front Immunol 3: 209, 2012.

33. Imai K, Morio T, Zhu Y, Jin Y, Itoh S, Kajiwara M, Yata J, Mizutani S, Ochs HD and Nonoyama S: Clinical course of patients with WASP gene mutations. Blood 103: 456-464, 2004.

34. Zhao Q, Jiang L, Yu J, Xiao J and Zhao X: Genotype and phenotype correlation of Wiskott-Aldrich syndrome: A report based on 24 Chinese patients. J Third Mil Med Univ 33: 1404-1407, 2011.

35. Welch MD, DePace AH, Verma S, Iwamatsu A and Mitchison TJ: The human Arp2/3 complex is composed of evolutionarily conserved subunits and is localized to cellular regions of dynamic actin filament assembly. J Cell Biol 138: 375-384, 1997.

36. Wu M, Katta A, Gadde MK, Liu H, Kakarla SK, Fannin J, Paturi S, Arvapalli RK, Rice KM, Wang Y and Blough ER: Aging-associated dysfunction of Akt/protein kinase B: S-nitrosylation and acetaminophen intervention. PLoS One 4: e6430, 2009.

37. Weed SA, Karginov AV, Schafer DA, Weaver AM, Kinley AW, Cooper JA and Parsons JT: Cortactin localization to sites of actin assembly in lamellipodia requires interactions with F-actin and the Arp2/3 complex. J Cell Biol 151: 29-40, 2000.

38. Di Ciano C, Nie Z, Szászi K, Lewis A, Uruno T, Zhan X, Rotstein OD, Mak A and Kapus A: Osmotic stress-induced remodeling of the cortical cytoskeleton. Am J Physiol Cell Physiol 283: C850-C865, 2002.

39. Latreille M, Laberge MK, Bourret G, Yamani L and Larose L: Deletion of Nck1 attenuates hepatic ER stress signaling and improves glucose tolerance and insulin signaling in liver of obese mice. Am J Physiol Endocrinol Metab 300: E423-E434, 2011.

40. Qadir MI, Parveen A and Ali M: Cdc42: Role in cancer management. Chem Biol Drug Des 86: 432-439, 2015.

41. Boztug K, Schmidt M, Schwarzer A, Banerjee PP, Díez IA, Dewey RA, Böhm M, Nowrouzi A, Ball CR, Glimm H, et al: Stem-cell gene therapy for the Wiskott-Aldrich syndrome. N Engl J Med 363: 1918-1927, 2010. 